
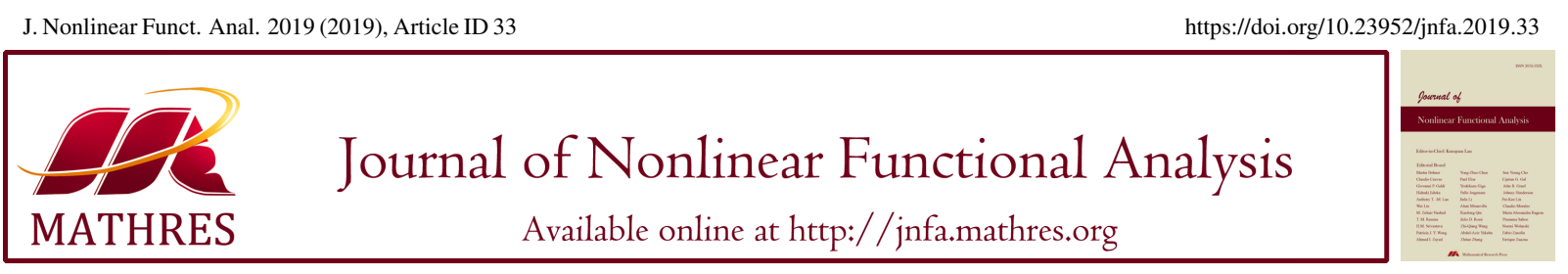

\title{
BOURBAKI COMPLETENESS IN QUASI METRIC SPACES
}

\author{
MERVE İLKHAN, EMRAH EVREN KARA* \\ Department of Mathematics, Faculty of Arts and Sciences, Düzce University, 81620, Düzce, Turkey
}

\begin{abstract}
The main purpose of this paper is to define a new type of boundedness in a quasi metric space. We introduce some new notions of completeness by clustering sequences belonging to the classes larger than the classes of Cauchy sequences in some sense. We also obtain some interesting results related to the compactness.
\end{abstract}

Keywords. Asymmetric norm; Boundedness; Compactness; Quasi metric.

2010 Mathematics Subject Classification. 54E50, 54E55.

\section{INTRODUCTION}

A metric is a distance function on a non-empty set and a metric space can be regarded as a structure on which functional analysis is developed. By modifying the definition of a metric, a new notion of a non-symmetric distance function is proposed in 1930's. Since the requirement of the symmetry property is omitted in this new definition, the classical theorems for metric spaces are no longer clear and some of them are not true for the resulting space which is called as a quasi metric space (also known as an asymmetric metric space). For example, compactness and sequentially compactness do not coincide in contrary to the metric case. Also, since a quasi metric generates three different topologies, many nonequivalent notions of completeness occur on such a space. The focus of this paper is on completeness, compactness and precompactness which are most used in functional analysis.

The concept of a quasi metric space introduced by Wilson [1] and many authors have studied extensively to improve the theory of quasi metric spaces. For some of them, we refer to the papers by Albert [2], Bodjanova [3], Doitchinov [4], Kelly [5], Künzi [6, 7, 8, 9], Reilly, Subrahmanyam and Vamanamurthy [10], Reilly and Vamanamurthy [11], Romaguera [12], Romaguera and Gutierrez [13], Stoltenberg [14] and the references therein. Quasi metrics are not only a subject of intensive research in the mathematical branches but also in some other branches of sciences such as computer science, material science, biology etc; see [15, 16, 17].

\footnotetext{
${ }^{*}$ Corresponding author.
}

E-mail addresses: merveilkhan@gmail.com (M. İlkhan), eevrenkara@ duzce.edu.tr (E.E. Kara).

Received March 17, 2019; Accepted July 18, 2019. 
In the theory of metric spaces, some classes of bounded sets are important such as the class of sets with finite diameter, the class of totally bounded sets (or equivalently precompact sets) and the class of Bourbaki bounded sets which are called as finitely chainable in [18]. The last type of boundedness appeared to characterize metric spaces on which every uniformly continuous function is bounded. As it is a known fact that totally boundedness of a set can be characterized by Cauchy sequences (see [19]). Garrido and Merono [20] characterized Bourbaki boundedness in terms of new sequences named as Bourbaki Cauchy and cofinally Bourbaki Cauchy. For more results related to these new concepts, one can see [21, 22]. By using these new general Cauchy sequences, the authors defined new types of completeness for metric spaces called as Bourbaki complete and cofinally Bourbaki complete. Since Bourbaki completeness or cofinally Bourbaki completeness is a property stronger than completeness but weaker than compactness, these new type complete spaces are important.

In a quasi metric space, the totally boundedness of a set implies that the set is also precompact. But there are precompact sets which are not totally bounded in a quasi metric space (see Example 3.1 in [13]). Also, we encounter with a new notion called as outside precompactness in a quasi metric space which is weaker than the precompactness. One of our main purposes is to define (outside) Bourbaki boundedness in a quasi metric space and examine the relations with (outside) precompactness and usual boundedness. Moreover, after defining Bourbaki Cauchy and cofinally Bourbaki Cauchy sequences in a quasi metric space, we investigate whether one can characterize Bourbaki boundedness in terms of those sequences. By clustering them, we define different types of completeness. Also, we give some results related to compactness, sequentially compactness and uniformly locally compactness in quasi metric spaces.

\section{PRELIMINARIES}

We begin this section with the definition of a quasi metric space. In the sequel, we present some basic concepts and results related to the general theory of quasi metric spaces.

A quasi metric $\rho$ is a distance function satisfying the following conditions on a non-empty set $X$ :

(1) $\rho(x, y) \geq 0$ for all $x, y \in X$,

(2) $\rho(x, x)=0$ for all $x \in X$,

(3) $\rho(x, y)=\rho(y, x)=0 \Rightarrow x=y$,

(4) $\rho(x, y) \leq \rho(x, z)+\rho(z, y)$ for all $x, y, z \in X$.

That is, it is possible $\rho(x, y) \neq \rho(y, x)$ for some $x, y \in X$. $(X, \rho)$ is said to be a quasi metric space. If the function $\rho$ satisfies the conditions (1), (2) and (4) only, it is called as a quasi pseudometric (semimetric). Then, $(X, \rho)$ is said to be a quasi pseudometric space.

The function $\bar{\rho}$ defined by $\bar{\rho}(x, y)=\rho(y, x)$ for all $x, y \in X$ is also a quasi (pseudo)metric on $X$ and called as the conjugate quasi (pseudo)metric of $\rho$. Also, the mapping $\rho^{s}(x, y)=\max \{\rho(x, y), \bar{\rho}(x, y)\}$ is a (pseudo)metric on $X$. The inequalities $\rho(x, y) \leq \rho^{s}(x, y)$ and $\bar{\rho}(x, y) \leq \rho^{s}(x, y)$ hold for these mappings.

For a quasi metric space $(X, \rho)$, the family of $\rho$-open balls $B_{\rho}(x, \varepsilon)=\{y \in X: \rho(x, y)<\varepsilon\}$ generates a topology $\tau_{\rho}$ on $X$. Similarly, one obtains two different topologies $\tau_{\bar{\rho}}$ and $\tau_{\rho^{s}}$ generated on $X$ by the families of $\bar{\rho}$-open balls and $\rho^{s}$-open balls, respectively. Note that the topology $\tau_{\rho}$ is $T_{0}$ but it is neither $T_{1}$ nor $T_{2}$ unlike a topology generated by a usual metric. This topology is $T_{1}$ if and only if $\rho(x, y)>0$ for distinct $x, y \in X$ (see [23] for more results). 
A sequence $\left(x_{n}\right)$ in a quasi metric space $(X, \rho)$ is said to be left (right) $\rho$-convergent to a point $x \in X$ if $\rho\left(x, x_{n}\right) \rightarrow 0\left(\rho\left(x_{n}, x\right) \rightarrow 0\right)$ as $n \rightarrow \infty$ ([1]). Throughout the paper, we briefly write " $\rho$-convergence" which means left $\rho$-convergence of a sequence in a quasi metric space $(X, \rho)$.

Firstly, Kelly [5] defined Cauchy sequences in a quasi metric space to prove Baire category theorem. He observed that a convergent sequence need not be Cauchy and he could not use this Cauchy definition to obtain a completion for a quasi metric space. Later, Reilly, Subrahmanyam and Vamanamurthy [10] defined some different types of Cauchy sequences to overcome these problems which generalize Kelly's definition.

A sequence $\left(x_{n}\right)$ in a quasi metric space $(X, \rho)$ is said to be:

(1) $\rho^{s}$-Cauchy if, for every $\varepsilon>0$, there exists $n_{0} \in \mathbb{N}$ such that $\rho\left(x_{n}, x_{m}\right)<\varepsilon$ for all $n, m \geq n_{0}$.

(2) left (right) $K$-Cauchy if, for every $\varepsilon>0$, there exists $n_{0} \in \mathbb{N}$ such that $\rho\left(x_{m}, x_{n}\right)<\varepsilon\left(\rho\left(x_{n}, x_{m}\right)<\right.$ $\varepsilon)$ for all $n \geq m \geq n_{0}$.

(3) weakly left (right) $K$-Cauchy if, for every $\varepsilon>0$, there exists $n_{0} \in \mathbb{N}$ such that $\rho\left(x_{n_{0}}, x_{n}\right)<\varepsilon$ $\left(\rho\left(x_{n}, x_{n_{0}}\right)<\varepsilon\right)$ for all $n \geq n_{0}$.

(4) left (right ) $\rho$-Cauchy if, for every $\varepsilon>0$, there exist $x \in X$ and $n_{0} \in \mathbb{N}$ such that $\rho\left(x, x_{n}\right)<\varepsilon$ $\left(\rho\left(x_{n}, x\right)<\varepsilon\right)$ for all $n \geq n_{0}$.

For the relations and converse examples related to these Cauchy sequences, we refer to [10]. Note that left (right) $\rho$-convergence of a sequence in a quasi metric space implies only that the sequence is left (right) $\rho$-Cauchy. Also, it was proved in $[3,11]$ that if a left $K$-Cauchy sequence has a left $\rho$-convergent subsequence, then the sequence itself is left $\rho$-convergent to the same point. But, this is not the case for weakly left $K$-Cauchy and left $\rho$-Cauchy sequences (see Example 4.1 in [13]).

By using left (right) $\rho$-convergence or convergence with respect to the metric $\rho^{s}$ of each of these seven type Cauchy sequence, many sequential completeness notions were defined for quasi metric spaces. A quasi metric space is called left $\rho$-complete ((weakly) left $K$-complete or $\rho^{s}$-complete) if every left $\rho$-Cauchy ((weakly) left $K$-Cauchy or $\rho^{s}$-Cauchy) sequence is left $\rho$-convergent. It is clear that left $\rho$-completeness implies weakly left $K$-completeness, weakly left $K$-completeness implies left $K$ completeness and left $K$-completeness implies $\rho^{s}$-completeness. On the other hand, it was proved in [12] that the notions of weakly left $K$-completeness and left $K$-completeness are equivalent.

In [24], the authors defined the reversibility $\lambda$ of a general metric space $(X, d)$ as

$$
\lambda=\sup _{\substack{x, y \in X \\ x \neq y}} \frac{d(x, y)}{d(y, x)} .
$$

By using this notion, they observed that left $K$-Cauchy and right $K$-Cauchy sequences coincide in a $T_{1}$ quasi metric space with finite reversibility. Also, they proved that corresponding completeness notions are equivalent.

By $\rho$-boundedness of a set $A$ in a quasi metric space $(X, \rho)$, we mean that there exist $x \in X$ and $r>0$ such that $A \subset B_{\rho}(x, r)$. Observe that this definition is not equivalent to the definition of boundedness of a set in a metric space. In a metric space, a set is bounded with respect to the given metric if the set has a finite diameter. Consider the quasi metric $\rho$ on the set of all real numbers $\mathbb{R}$ defined by $\rho(x, y)=$ $\max \{y-x, 0\}$. Then, the set $A=(-\infty, 0)$ is $\rho$-bounded since we have $A \subset B_{\rho}(0,1)=(-\infty, 1)$. Suppose that there exists a real number $M>0$ such that $\rho(x, y)<M$ for all $x, y \in A$. Choose $x=-(M+2)$, $y=-1$ in $A$. Then, we have $\rho(x, y)=M+1 \nless M$ which is a contradiction. Hence, it follows that 
$\operatorname{diam}(A)=\sup \{\rho(x, y): x, y \in A\}$ is not finite. In fact, $\operatorname{diam}(A)$ is the diameter of $A$ with respect to the metric $\rho^{s}$. We conclude that if a subset in a quasi metric space $(X, \rho)$ is bounded with respect to the metric $\rho^{s}$, then it is $\rho$-bounded from the fact that $B_{\rho^{s}}(x, r) \subset B_{\rho}(x, r)$ for all $x \in X$ and $r>0$. One can see from the above example that the converse statement is not true.

A subset $Y$ in a quasi metric space $(X, \rho)$ is said to be (outside) precompact if, for every $\varepsilon>0$, there exists a finite subset $F$ of $Y(X)$ such that $Y \subset \bigcup_{z \in F} B_{\rho}(z, \varepsilon)$. Unless otherwise stated, by (sequentially) compactness of a set in a quasi metric space, we mean that it is (sequentially) compact with respect to the topology generated by the given quasi metric.

A real valued nonnegative function $p$ on $X$ is called as an asymmetric norm if it satisfies the following axioms:

(1) $p(x)=p(-x)=0$ if and only if $x=0$,

(2) $p(\alpha x)=\alpha p(x)$

(3) $p(x+y) \leq p(x)+p(y)$,

for all $x, y \in X$ and $\alpha>0$. Similarly, conjugate asymmetric norm $\bar{p}$ on $X$ is defined by $\bar{p}(x)=p(-x)$ and also $p^{s}(x)=\max \{p(x), \bar{p}(x)\}$ becomes a norm on $X$.

By the analogue way that a norm generates a metric, an asymmetric norm $p$ on a vector space $X$ generates a quasi metric through the equality $\rho_{p}(x, y)=p(y-x)$ for all $x, y \in X$. Hence, the basic concepts defined for quasi metric spaces can be given similarly for asymmetric metric spaces. One can consult to the papers $[25,26,27,28]$ related to the theory of asymmetric normed spaces. Also, we point out that this theory has been improved by applying many problems in theoretical computer science and approximation theory (see for instance [29] and [30, 31], respectively).

\section{MAin Results}

We say that a point $y$ in a quasi metric space $(X, \rho)$ can be joined to the point $x$ with an $\varepsilon$-chain of length $m$ if there are points $a_{1}, a_{2}, \ldots, a_{m-1}$ in $X$ satisfying $\rho\left(x, a_{1}\right)<\varepsilon, \rho\left(a_{1}, a_{2}\right)<\varepsilon, \ldots, \rho\left(a_{m-1}, y\right)<\varepsilon$ and we denote the set of all such points $y$ in $X$ by $B_{\rho}^{m}(x, \varepsilon)$. The $\varepsilon$-enlargement of a subset $A$ in $X$ is given by

$$
A^{\varepsilon}=\bigcup\left\{B_{\rho}(x, \varepsilon): x \in A\right\}
$$

and the $m^{\text {th }} \varepsilon$-enlargement of the $\rho$-open ball $B_{\rho}(x, \varepsilon)$ is defined as

$$
B_{\rho}^{m}(x, \varepsilon)=\left(B_{\rho}^{m-1}(x, \varepsilon)\right)^{\varepsilon}=\bigcup\left\{B_{\rho}(y, \varepsilon): y \in B_{\rho}^{m-1}(x, \varepsilon)\right\}
$$

for $m \geq 2$.

Definition 3.1. A subset $Y$ in a quasi metric space $(X, \rho)$ is said to be $\rho$-Bourbaki bounded if for every $\varepsilon>0$ there exist $m \in \mathbb{N}$ and a finite subset $F$ of $Y$ such that

$$
Y \subset \bigcup\left\{B_{\rho}^{m}(x, \varepsilon): x \in F\right\}
$$

In this definition, if $F$ is a finite subset of $X$, then $Y$ is called as outside $\rho$-Bourbaki bounded.

In a metric space $(X, d)$, this two concepts are equivalent since we have $B_{d}^{m}(x, \varepsilon) \subset B_{d}^{2 m}(z, \varepsilon)$, where $z \in B_{d}^{m}(x, \varepsilon)$. However, this inclusion is not valid for quasi metric spaces. For example, let $X=[0,1]$ and

$$
\rho(x, y)=\left\{\begin{array}{cc}
y-x, & \text { if } x \leq y \\
1, & \text { if } x>y
\end{array}\right.
$$


Then, it is obvious that $B_{\rho}\left(0, \frac{1}{2}\right)=\left[0, \frac{1}{2}\right)$ is not contained in $B_{\rho}^{2}\left(\frac{1}{4}, \frac{1}{2}\right)=\left[\frac{1}{4}, 1\right]$. We have the following Lemma for quasi metric spaces.

Lemma 3.2. The inclusion

$$
B_{\rho}^{m}(x, \varepsilon) \subset B_{\rho}^{2 m}(z, \varepsilon)
$$

holds for every $z \in B_{\bar{\rho}}^{m}(x, \varepsilon)$.

Proof. Let $y \in B_{\rho}^{m}(x, \varepsilon)$. Then there exist $a_{1}, a_{2}, \ldots, a_{m-1} \in X$ such that $\rho\left(x, a_{1}\right)<\varepsilon, \rho\left(a_{1}, a_{2}\right)<\varepsilon, \ldots, \rho\left(a_{m-1}, y\right)<$ $\varepsilon$. Also, by hypothesis we have $\bar{\rho}\left(x, b_{1}\right)<\varepsilon, \bar{\rho}\left(b_{1}, b_{2}\right)<\varepsilon, \ldots, \bar{\rho}\left(b_{m-1}, z\right)<\varepsilon$ for some points $b_{1}, b_{2}, \ldots, b_{m-1} \in$ $X$. By using these points with $x$, we can join $y$ to $z$ by means of an $\varepsilon$-chain of length $2 m$ with respect to quasi metric $\rho$. This implies that $y \in B_{\rho}^{2 m}(z, \varepsilon)$.

In an asymmetric normed space $(X, p)$, these notions can be defined in the same way. On the contrary to quasi metric spaces, the equality $B_{p}(x, m r)=B_{p}^{m}(x, r)$ holds for every $x \in X, m \in \mathbb{N}$ and $r>0$. It can be shown that the inclusion $B_{p}(x, m r) \subset B_{p}^{m}(x, r)$ is satisfied while the reverse inclusion is obvious. If we choose $y \in B_{p}(x, m r)$, then $p\left(a_{i+1}-a_{i}\right)=\frac{1}{m} p(y-x)<r$, where $a_{i}=\frac{m-i}{m} x+\frac{i}{m} y$ for $i=0, \ldots, m$. This implies that $y$ can be joined to $x$ with an $\varepsilon$-chain of length $m$, that is, $y \in B_{p}^{m}(x, r)$. Thus, the concepts of (outside) $p$-Bourbaki boundedness and $p$-boundedness are equivalent in an asymmetric normed space.

As a consequence of the inclusion $B_{\rho}^{m}(x, \varepsilon) \subset B_{\rho}(x, m \varepsilon)$, we can say that every (outside) $\rho$-Bourbaki bounded set in a quasi metric space is $\rho$-bounded. From the next example, it is observed that the converse fails.

Example 3.3. Consider the space of all real numbers $\mathbb{R}$ with the quasi metric $\tilde{\rho}(x, y)=\min \{1, \rho(x, y)\}$, where $\rho(x, y)=\max \{y-x, 0\}$. Then $\mathbb{N}$ is $\tilde{\rho}$-bounded subset of $\mathbb{R}$. Indeed, for $r>1$ and $x \in \mathbb{R}$, we have $\mathbb{N} \subset B_{\tilde{\rho}}(x, r)=\mathbb{R}$. But, if we choose $\varepsilon \leq 1$, since for any $x \in \mathbb{R}$ and $m \in \mathbb{N}$, we have $B_{\tilde{\rho}}^{m}(x, \varepsilon)=$ $(-\infty, x+m \varepsilon)$. We cannot cover $\mathbb{N}$ with finitely many of these sets. Hence $\mathbb{N}$ is not outside $\tilde{\rho}$-Bourbaki bounded and so it is also not $\tilde{\rho}$-Bourbaki bounded.

By definitions, it is clear that every (outside) precompact set in a quasi metric space is (outside) $\rho$ Bourbaki bounded and the reverse statement is not true in general as shown in the following example.

Example 3.4. Consider the space of all bounded sequences $\ell_{\infty}$ with the quasi metric $\rho(x, y)=\sup _{i \in \mathbb{N}}\left(y_{i}-\right.$ $\left.x_{i}\right)^{+}$, where $x=\left(x_{i}\right), y=\left(y_{i}\right) \in \ell_{\infty}$ and $\left(y_{i}-x_{i}\right)^{+}=\max \left\{y_{i}-x_{i}, 0\right\}$ for all $i \in \mathbb{N}$. Let $x^{n}$ be the sequence whose first $n$ terms are equal to 1 and other infinitely many terms are equal to zero, that is, $x^{n}=(1,1, \ldots, 1,0,0, \ldots)$ for all $n \in \mathbb{N}$. Put $Y=\left\{x^{n}: n \in \mathbb{N}\right\} \subset \ell_{\infty}$. Whereas it was proved in [25] that this set is not precompact, $Y$ is $\rho$-Bourbaki bounded since given any $\varepsilon>0$. We have $Y \subset B_{\rho}^{m}\left(x^{1}, \varepsilon\right)$, where $m \varepsilon>1$.

As it is known, outside precompactness is strictly weaker than precompactness in a quasi metric space. This is true for outside $\rho$-Bourbaki boundedness and $\rho$-Bourbaki boundedness. That is, every $\rho$-Bourbaki bounded set is outside $\rho$-Bourbaki bounded. For the converse statement, we need an extra condition.

Example 3.5. Let $X=[0,1]$ and $\rho$ be the quasi metric on $X$ defined as

$$
\rho(x, y)=\left\{\begin{array}{cc}
y-x, & \text { if } x \leq y \\
1, & \text { if } x>y .
\end{array}\right.
$$


Then the set $Y=(0,1)$ is outside $\rho$-Bourbaki bounded by the fact that $Y \subset B_{\rho}^{m}(0, \varepsilon)$, where $m>1 / \varepsilon$.

Suppose that $Y$ is also $\rho$-Bourbaki bounded. Then, given any $\varepsilon \leq 1$, for finitely many points $x_{1}, \ldots, x_{n}$ in $Y, Y$ must be covered by the union of $B_{\rho}^{m}\left(x_{i}, \varepsilon\right)(i=1, \ldots, n)$ for some $m \in \mathbb{N}$. But in this case, the union $\bigcup_{i=1}^{n} B_{\rho}^{m}\left(x_{i}, \varepsilon\right)$ equals to the set $[a, b+m \varepsilon)$, where $a=\min \left\{x_{i}: i=1, \ldots, n\right\}$ and $b=\max \left\{x_{i}: i=1, \ldots, n\right\}$. This is a contradiction since the set $[a, b+m \varepsilon)$ cannot contain $Y$. Thus, $Y$ is not $\rho$-Bourbaki bounded.

Theorem 3.6. A subset $Y$ in a quasi metric space $(X, \rho)$ is $\rho$-Bourbaki bounded if and only if for every $\varepsilon>0$ there exist $m \in \mathbb{N}$ and $\left\{x_{1}, x_{2}, \ldots, x_{n}\right\} \subset X$ such that $Y \subset \cup_{i=1}^{n} B_{\rho}^{m}\left(x_{i}, \varepsilon\right)$ and $Y \cap B_{\bar{\rho}}^{m}\left(x_{i}, \varepsilon\right) \neq \emptyset$ for $i=1, \ldots n$.

Proof. The necessity is obvious. Now assume that the sufficiency of the condition stated in the theorem holds. Choose $y_{i} \in Y \cap B_{\bar{\rho}}^{m}\left(x_{i}, \varepsilon\right)$ for all $i=1, \ldots n$. Using Lemma 3.2, we obtain the following inclusion

$$
Y \subset \bigcup_{i=1}^{n} B_{\rho}^{m}\left(x_{i}, \varepsilon\right) \subset \bigcup_{i=1}^{n} B_{\rho}^{2 m}\left(y_{i}, \varepsilon\right),
$$

which proves that $Y$ is $\rho$-Bourbaki bounded.

Bourbaki boundedness of a set with respect to the metric $\rho^{s}$ implies that it is both $\rho$-Bourbaki bounded and $\bar{\rho}$-Bourbaki bounded. The following example shows that the converse statement is not true.

Example 3.7. Consider the quasi metric space $(X, \rho)$ in Example 3.5. Let $x$ be an arbitrary point in $X$. Given $\varepsilon>0$, choose $m \in \mathbb{N}$ such that $\frac{1}{m}<\varepsilon$. Put $a_{i}=\frac{i x}{m}(i=0, \ldots, m)$. Then, $\rho\left(a_{i}, a_{i+1}\right)=\frac{x}{m}<\varepsilon$ for $i=0, \ldots, m-1$. Hence any point $x$ in $X$ can be joined to zero by an $\varepsilon$-chain of length $m$. This means that $X=B_{\rho}^{m}(0, \varepsilon)$ and $X$ is $\rho$-Bourbaki bounded. In a similar way, it can be shown that $X=B_{\bar{\rho}}^{m}(1, \varepsilon)$ and then $X$ is $\bar{\rho}$-Bourbaki bounded. However, $X$ is not $\rho^{s}$-Bourbaki bounded since $\rho^{s}$ is the discrete metric on $X$.

The following theorem gives the relations related to Bourbaki boundedness of a space with respect to quasi metrics $\rho, \bar{\rho}$ and metric $\rho^{s}$ under some assumptions.

Theorem 3.8. In a $T_{1}$ quasi metric space $(X, \rho)$, if $\lambda=\sup _{\substack{x, y \in X \\ x \neq y}} \frac{\rho(x, y)}{\rho(y, x)}$ exists, then the following statements are equivalent

(1) $X$ is $\rho$-Bourbaki bounded,

(2) $X$ is $\bar{\rho}$-Bourbaki bounded,

(3) $X$ is $\rho^{s}$-Bourbaki bounded.

Proof. $1 \Rightarrow 2$ If $X$ is $\rho$-Bourbaki bounded, then there exist $m \in \mathbb{N}$ and a finite subset $F$ of $X$ such that

$$
X=\bigcup_{z \in F} B_{\rho}^{m}\left(z, \frac{\varepsilon}{\lambda}\right) .
$$

By the hypothesis, inequality $\bar{\rho}(x, y) \leq \lambda \rho(x, y)$ holds for every $x, y \in X$ with $x \neq y$. Hence,

$$
B_{\rho}^{m}\left(z, \frac{\varepsilon}{\lambda}\right) \subset B_{\bar{\rho}}^{m}(z, \varepsilon)
$$

for all $z \in F$, which implies that $X=\bigcup_{z \in F} B_{\bar{\rho}}^{m}(z, \varepsilon)$. So, $X$ is $\bar{\rho}$-Bourbaki bounded.

$2 \Rightarrow 3$ It can be easily seen that, for a finite subset $F$ of $X$ and $m \in \mathbb{N}$,

$$
X=\bigcup_{z \in F} B_{\bar{\rho}}^{m}\left(z, \frac{\varepsilon}{M}\right)=\bigcup_{z \in F} B_{\rho^{s}}^{m}(z, \varepsilon)
$$


where $M=\max \{1, \lambda\}$.

$3 \Rightarrow 1$ It is clear since the inclusion

$$
B_{\rho^{s}}^{m}(x, \varepsilon) \subset B_{\rho}^{m}(x, \varepsilon)
$$

holds for all $x \in X$ and $m \in \mathbb{N}$.

Remark 3.9. Unlike metric spaces, in quasi metric spaces there are $\rho$-Bourbaki bounded sets whose subsets and also closures with respect to the topology generated by the quasi metric may not be $\rho$ Bourbaki bounded.

Example 3.10. Let $(X, \rho)$ be the quasi metric space in Example 3.5. Then the set $Y=\{0\} \cup\left\{\frac{1}{n}: n \in \mathbb{N}\right\}$ is $\rho$-Bourbaki bounded due to the fact that $Y \subset B_{\rho}^{m}(0, \varepsilon)=[0, m \varepsilon)$, where $m>1 / \varepsilon$. However, the subset $\left\{\frac{1}{n}: n \in \mathbb{N}\right\}$ of $Y$ is not $\rho$-Bourbaki bounded since it cannot be covered by the union of finitely many sets $B_{\rho}^{m}\left(\frac{1}{n}, \varepsilon\right)=\left[\frac{1}{n}, \frac{1}{n}+m \varepsilon\right)$.

Example 3.11. Consider $\mathbb{R}$ with the quasi metric $\rho(x, y)=\max \{y-x, 0\}$. Since, given any $\varepsilon>0$, the inclusion $(-\infty, 1] \subset B_{\rho}(1, \varepsilon)=(-\infty, 1+\varepsilon)$ holds, the set $(-\infty, 1]$ is $\rho$-Bourbaki bounded. But the closure with respect to the topology generated by $\rho, \tau_{\rho}-\mathrm{Cl}((-\infty, 1])=\mathbb{R}$ is not $\rho$-Bourbaki bounded. Indeed, it cannot be covered by finitely many $B_{\rho}^{m}(x, \varepsilon)=(-\infty, x+m \varepsilon)$ for any $x \in \mathbb{R}$ and $m \in \mathbb{N}$.

Theorem 3.12. A subset $Y$ in a quasi metric space $(X, \rho)$ is (outside) $\rho$-Bourbaki bounded if and only if the closure of $Y$ with respect to the topology generated by the conjugate quasi metric $\bar{\rho}, \tau_{\bar{\rho}}-\mathrm{Cl}(Y)$ is (outside) $\rho$-Bourbaki bounded.

Proof. Let $Y$ be $\rho$-Bourbaki bounded subset of $X$. Then, given any $\varepsilon>0$, we can find a finite subset $F \subset Y$ and $m \in \mathbb{N}$ satisfying $Y \subset \bigcup\left\{B_{\rho}^{m}(x, \varepsilon): x \in F\right\}$. If $z$ is any point in $\tau_{\bar{\rho}-}-\mathrm{Cl}(Y)$, then there exist some points $y$ in $Y$ such that $\bar{\rho}(z, y)<\varepsilon$. By hypothesis, $y$ belongs to $B_{\rho}^{m}(x, \varepsilon)$ for at least one $x \in F$. Hence we obtain an $\varepsilon$-chain of length $m+1$ from $x$ to $z$. It follows that $\tau_{\bar{\rho}}-\mathrm{Cl}(Y) \subset \bigcup\left\{B_{\rho}^{m+1}(x, \varepsilon): x \in F\right\}$. So, $\tau_{\bar{\rho}}-\mathrm{Cl}(Y)$ is $\rho$-Bourbaki bounded.

Conversely, we suppose that $\tau_{\bar{\rho}}-\mathrm{Cl}(Y) \subset \bigcup_{i=1}^{n} B_{\rho}^{m}\left(z_{i}, \varepsilon\right)$, where $z_{i} \in \tau_{\bar{\rho}}-\mathrm{Cl}(Y)$ for $i=1, \ldots, n$ and $m \in \mathbb{N}$. Then, $B_{\bar{\rho}}\left(z_{i}, \varepsilon\right) \cap Y \neq \emptyset$. So, we can choose $y_{i} \in B_{\bar{\rho}}^{m}\left(z_{i}, \varepsilon\right) \cap Y$ for $i=1, \ldots, n$. By Lemma 3.2, the inclusion $B_{\rho}^{m}\left(z_{i}, \varepsilon\right) \subset B_{\rho}^{2 m}\left(y_{i}, \varepsilon\right)$ holds. Consequently, $Y$ can be covered by the union of $B_{\rho}^{2 m}\left(y_{i}, \varepsilon\right)$, which means that $Y$ is $\rho$-Bourbaki bounded.

Now, we define some type of Bourbaki Cauchy sequences in a quasi metric space.

Definition 3.13. Let $(X, \rho)$ be a quasi metric space. A sequence $\left(x_{n}\right)$ in $X$ is said to be left (right) $\rho$-Bourbaki Cauchy if, for every $\varepsilon>0$, there exist $n_{0} \in \mathbb{N}, m \in \mathbb{N}$ and $x \in X$ such that $x_{n} \in B_{\rho}^{m}(x, \varepsilon)$ $\left(x_{n} \in B_{\bar{\rho}}^{m}(x, \varepsilon)\right)$ for all $n \geq n_{0}$. It is said to be weakly left (right) $K$-Bourbaki Cauchy if, for every $\varepsilon>0$, there exist $n_{0} \in \mathbb{N}$ and $m \in \mathbb{N}$ such that $x_{n} \in B_{\rho}^{m}\left(x_{n_{0}}, \mathcal{\varepsilon}\right)\left(x_{n} \in B_{\bar{\rho}}^{m}\left(x_{n_{0}}, \mathcal{\varepsilon}\right)\right)$ for all $n \geq n_{0}$. It is said to be $\rho^{s}$-Bourbaki Cauchy if, for every $\varepsilon>0$, there exist $n_{0} \in \mathbb{N}, m \in \mathbb{N}$ and $x \in X$ such that $x_{n} \in B_{\rho^{s}}^{m}(x, \varepsilon)$ for all $n \geq n_{0}$.

Definition 3.14. Let $(X, \rho)$ be a quasi metric space. A sequence $\left(x_{n}\right)$ in $X$ is said to be left (right) $\rho$ cofinally Bourbaki Cauchy if, for every $\varepsilon>0$, there exist $m \in \mathbb{N}, x \in X$ and an infinite subset $\mathbb{N}_{\varepsilon}$ of $\mathbb{N}$ such that $x_{n} \in B_{\rho}^{m}(x, \varepsilon)\left(x_{n} \in B_{\bar{\rho}}^{m}(x, \varepsilon)\right)$ for all $n \in \mathbb{N}_{\varepsilon}$. It is said to be weakly left (right) $K$-cofinally Bourbaki Cauchy if, for every $\varepsilon>0$, there exist $m, n_{0} \in \mathbb{N}$ and an infinite subset $\mathbb{N}_{\varepsilon}$ of $\mathbb{N}$ such that $x_{n} \in B_{\rho}^{m}\left(x_{n_{0}}, \varepsilon\right)$ 
$\left(x_{n} \in B_{\bar{\rho}}^{m}\left(x_{n_{0}}, \varepsilon\right)\right)$ for all $n \in \mathbb{N}_{\varepsilon}$. It is said to be $\rho^{s}$-cofinally Bourbaki Cauchy if, for every $\varepsilon>0$, there exist $m \in \mathbb{N}, x \in X$ and an infinite subset $\mathbb{N}_{\varepsilon}$ of $\mathbb{N}$ such that $x_{n} \in B_{\rho^{s}}^{m}(x, \varepsilon)$ for all $n \in \mathbb{N}_{\varepsilon}$.

From this definition, it is obvious that the class of right $\rho$-(cofinally) Bourbaki Cauchy sequences is equivalent to the class of left $\bar{\rho}$-(cofinally) Bourbaki Cauchy sequences and the class of weakly right $K$-(cofinally) Bourbaki Cauchy sequences with respect to the quasi metric $\rho$ is equivalent to the class of weakly left $K$-(cofinally) Bourbaki Cauchy sequences with respect to the quasi metric $\bar{\rho}$.

In a quasi metric space, while a weakly left $K$-Bourbaki Cauchy sequence is left $\rho$-Bourbaki Cauchy, the converse implication is not true.

Example 3.15. Let $X=[0,1]$ and $\rho$ be the quasi metric on $X$ defined as

$$
\rho(x, y)= \begin{cases}0, & \text { if } x \leq y \\ 1, & \text { if } x>y .\end{cases}
$$

Given any sequence $\left(x_{n}\right)$ in $X$, we have $\rho\left(0, x_{n}\right)=0$ for all $n \in \mathbb{N}$, which implies that the sequence $\left(x_{n}\right)$ is $\rho$-convergent to 0 . Then, every sequence in this space is left $\rho$-Bourbaki Cauchy.

On the other hand, the sequence $\left(\frac{1}{2^{n}}\right)$ is not weakly left $K$-Bourbaki Cauchy since there are infinitely many terms outside of $B_{\rho}^{m}\left(\frac{1}{2^{n}}, \varepsilon\right)=\left[\frac{1}{2^{n}}, 1\right]$ for all $n, m \in \mathbb{N}$.

In a quasi metric space, while a weakly left $K$-cofinally Bourbaki Cauchy sequence is left $\rho$-cofinally Bourbaki Cauchy, the converse implication is not true. The following example also shows that there exists a weakly left $K$-cofinally Bourbaki Cauchy (left $\rho$-cofinally Bourbaki Cauchy) sequence in a quasi metric space which is not weakly left $K$-Bourbaki Cauchy (left $\rho$-Bourbaki Cauchy).

Example 3.16. Let $X=\mathbb{R}$ and $\rho$ be the quasi metric on $X$ defined in Example 3.5. It is clear that the sequence $\left(x_{n}\right)=\left(1, \frac{1}{2}, 3, \frac{1}{4}, 5, \frac{1}{6}, \ldots\right)$ is a left $\rho$-cofinally Bourbaki Cauchy sequence but it is not weakly left $K$-cofinally Bourbaki Cauchy. The sequence $\left(y_{n}\right)=(0,1,0,2,0,3, \ldots)$ is not a left $\rho$-Bourbaki Cauchy sequence and so it is not a weakly left $K$-Bourbaki Cauchy sequence. In fact, given any $m, n \in \mathbb{N}$ and $x \in X$, there exits an even number $n_{1}$ with $n_{1}>n$ such that $x+m<\frac{n_{1}}{2}$, which means that $y_{n_{1}} \notin B^{m}(x, 1)=[x, x+m)$. But, it is a weakly left $K$-cofinally Bourbaki Cauchy sequence and so it is left $\rho$-cofinally Bourbaki Cauchy. To observe this fact, take $m=n_{0}=1$ and $\mathbb{N}_{\varepsilon}=\{1,3,5,7, \ldots\}$. Hence, given any $\varepsilon>0$, we have $y_{n} \in B_{\rho}(0, \varepsilon)=[0, \varepsilon)$ for all $n \in \mathbb{N}_{\varepsilon}$.

The following example shows that there is a left $\rho$-Bourbaki Cauchy sequence in a quasi metric space which is not left $\rho$-Cauchy. Also, it is an example of a weakly left $K$-Bourbaki Cauchy sequence. From Example 3.15, we have a left $\rho$-Cauchy sequence, which is not weakly left $K$-Bourbaki Cauchy. Hence, we conclude that there is no relation between weakly left $K$-Bourbaki Cauchy sequences and left $\rho$ Cauchy sequences.

Example 3.17. Let $(X, \rho)$ be the quasi metric space in the previous example and let $\left(x_{n}\right)$ be the sequence of some enumeration of the set $\mathbb{Q} \cap[0,1]$. Given any $\varepsilon>0$, all terms of the sequence are contained in $B_{\rho}^{m}(0, \varepsilon)=[0,1]$, where $m>1 / \varepsilon$. This means that $\left(x_{n}\right)$ is a weakly left $K$-Bourbaki Cauchy sequence and more generally it is a left $\rho$-Bourbaki Cauchy sequence. This sequence cannot be a left $\rho$-Cauchy sequence since for $\varepsilon=1 / 2$ and for any $x \in X$, there are infinitely many terms of the sequence which are not contained in $B_{\rho}(x, 1 / 2)=[x, x+1 / 2)$. 
If $\left(x_{n}\right)$ is a (cofinally) Bourbaki Cauchy sequence with respect to the metric $\rho^{s}$, then it is left $\rho$ (cofinally) Bourbaki (weakly left $K$-(cofinally) Bourbaki) Cauchy and right $\rho$-(cofinally) Bourbaki (weakly right $K$-(cofinally) Bourbaki) Cauchy, since the inclusions

$$
B_{\rho^{s}}^{m}(x, \varepsilon) \subset B_{\rho}^{m}(x, \varepsilon) \text { and } B_{\rho^{s}}^{m}(x, \varepsilon) \subset B_{\bar{\rho}}^{m}(x, \varepsilon)
$$

hold for every $\varepsilon>0, m \in \mathbb{N}$ and $x \in X$. Being both left $\rho$-(cofinally) Bourbaki (weakly left $K$-(cofinally) Bourbaki) Cauchy and right $\rho$-(cofinally) Bourbaki (weakly right $K$-(cofinally) Bourbaki) Cauchy sequence in a quasi metric space does not imply that the sequence is (cofinally) Bourbaki Cauchy sequence with respect to the metric $\rho^{s}$.

Example 3.18. Let $(X, \rho)$ be the quasi metric space in Example 3.5. Consider the sequence $(0,1,0,1, \ldots)$ in $X$. Given any $\varepsilon>0$, there exists $m \in \mathbb{N}$ such that $\frac{1}{m}<\varepsilon$. Then all terms of the sequence is contained in $B_{\rho}^{m}(0, \varepsilon)$ and $B_{\bar{\rho}}^{m}(1, \varepsilon)$, which imply respectively that the sequence is weakly left $K$-(cofinally)Bourbaki Cauchy and weakly right $K$-(cofinally)Bourbaki Cauchy. But this sequence is neither a Bourbaki Cauchy sequence nor a cofinally Bourbaki Cauchy sequence with respect to the metric $\rho^{s}$ which is discrete metric on $X$. Indeed, $m^{\text {th }} \varepsilon$-enlargement of any $\rho^{s}$-open ball in $X$ consists of exactly one point for $\varepsilon \leq 1$.

We have the following theorem which can be proved as Theorem 3.8.

Theorem 3.19. In a $T_{1}$ quasi metric space $(X, \rho)$, if $\lambda=\sup _{\substack{x, y \in X \\ x \neq y}} \frac{\rho(x, y)}{\rho(y, x)}$ exists, then the following statements are equivalent.

(1) $\left(x_{n}\right)$ is a left $\rho$-Bourbaki (weakly left $K$-Bourbaki) Cauchy sequence.

(2) $\left(x_{n}\right)$ is a right $\rho$-Bourbaki (weakly right $K$-Bourbaki) Cauchy sequence.

(3) $\left(x_{n}\right)$ is a $\rho^{s}$-Bourbaki Cauchy sequence.

In the following example, we observe that a weakly left $K$-(cofinally) Bourbaki Cauchy or left $\rho$ (cofinally) Bourbaki Cauchy sequence does not imply that the sequence is also a weakly right $K$ (cofinally) Bourbaki Cauchy or right $\rho$-(cofinally) Bourbaki Cauchy sequence and vice versa.

Example 3.20. Let $X=(0,1)$ and $\rho$ be the quasi metric on $X$ defined as

$$
\rho(x, y)=\left\{\begin{array}{cc}
x-y, & \text { if } y \leq x, \\
1, & \text { if } y>x .
\end{array}\right.
$$

The sequence $\left(\frac{1}{n+1}\right)$ is weakly left $K$-Bourbaki Cauchy but it is not right $\rho$-cofinally Bourbaki Cauchy. In fact, given any $\varepsilon>0$, choose $n_{0} \in \mathbb{N}$ with $\frac{1}{n_{0}}<\varepsilon$ and $m=1$. Hence, $x_{n} \in B_{\rho}^{m}\left(x_{n_{0}}, \varepsilon\right)=\left(0, x_{n_{0}}\right]$. Also, given any $x \in X, m \in \mathbb{N}$ and any infinite subset $\mathbb{N}_{1}$ of $\mathbb{N}$, there exists $n \in \mathbb{N}_{1}$ such that $\frac{1}{n+1} \notin B_{\bar{\rho}}^{m}(x, 1)=$ $[x, 1)$.

From the definitions, the following results can be seen easily.

Corollary 3.21. Let $\left(x_{n}\right)$ be a sequence in $(X, \rho)$.

(1) If $\left(x_{n}\right)$ is weakly left $K$-Bourbaki Cauchy sequence in $X$, then the set $\left\{x_{n}: n \in \mathbb{N}\right\}$ is $\rho$-Bourbaki bounded.

(2) If $\left(x_{n}\right)$ is $\rho$-convergent to $x$, then the set $\left\{x_{n}: n \in \mathbb{N}\right\}$ is outside $\rho$-Bourbaki bounded and the set $\{x\} \cup\left\{x_{n}: n \in \mathbb{N}\right\}$ is $\rho$-Bourbaki bounded. 
On the other hand, in a quasi metric space, there are $\rho$-convergent sequences which are not $\rho$-Bourbaki bounded. For example, using the quasi metric space in Example 3.5, we observe that the sequence $\left(\frac{1}{n}\right)$ is $\rho$-convergent to zero but the set $\left\{\frac{1}{n}: n \in \mathbb{N}\right\}$ is not $\rho$-Bourbaki bounded which is proved in Example 3.10 .

Theorem 3.22. Let $(X, \rho)$ be a quasi metric space. If $X$ is $\rho$-Bourbaki bounded, then every sequence in $X$ has a left $\rho$-Bourbaki Cauchy subsequence.

Proof. Suppose that $X$ is $\rho$-Bourbaki bounded. Let $\left(x_{n}\right)$ be an arbitrary sequence in $X$. There exist a finite subset $F_{1}$ of $X$ and $m_{1} \in \mathbb{N}$ such that $X=\cup\left\{B_{\rho}^{m_{1}}(x, 1 / 2): x \in F_{1}\right\}$. Then, for some $x_{1} \in F_{1}, B_{\rho}^{m_{1}}\left(x_{1}, 1 / 2\right)$ contains infinitely many terms of the sequence $\left(x_{n}\right)$. Let $M_{1}=\left\{n_{1}^{1}<n_{2}^{1}<\ldots<n_{i}^{1}<\ldots\right\} \subset \mathbb{N}$ be the set of indices of these terms and let $\left(x_{n_{i}^{1}}\right)$ be the subsequence of $\left(x_{n}\right)$. By continuing this process, we can find a finite subset $F_{k}$ of $X, m_{k} \in \mathbb{N}$ and $M_{k}=\left\{n_{1}^{k}<n_{2}^{k}<\ldots<n_{i}^{k}<\ldots\right\} \subset M_{k-1}$ such that, for all $i \in \mathbb{N}$, $x_{n_{i}^{k}} \in B_{\rho}^{m_{k}}\left(x_{k}, 1 / 2^{k}\right)$, where $x_{k} \in F_{k}$.

Hence, we conclude that the diagonal sequence $\left(x_{n_{i}^{i}}\right)$ is the desired left $\rho$-Bourbaki Cauchy subsequence. Indeed, given any $\varepsilon>0$, there exits $k_{\varepsilon} \in \mathbb{N}$ such that $\frac{1}{2^{k_{\varepsilon}}}<\varepsilon$. Thus $x_{n_{i}^{i}} \in B_{\rho}^{m_{k_{\varepsilon}}}\left(x_{k_{\varepsilon}}, \varepsilon\right)$ for every $i>k_{\varepsilon}$.

In [6], the author gave an example of a quasi metric space which is sequentially compact but not compact. Also, this is an example of a sequentially compact quasi metric space that is not precompact. We also observe that this space is not $\rho$-Bourbaki bounded. But sequentially compactness implies that every sequence in this space has a left $\rho$-Bourbaki Cauchy subsequence. Hence, we conclude that the converse assertion in the last theorem is not true. For the converse, we need an extra condition stated in the following theorem.

Theorem 3.23. Let $(X, \rho)$ be a quasi metric space. If $X$ is countable and every sequence in $X$ has a left $\rho$-Bourbaki Cauchy subsequence, then $X$ is $\rho$-Bourbaki bounded.

Proof. Let $X=\left\{x_{n}: n \in \mathbb{N}\right\}$ and assume that $X$ is not $\rho$-Bourbaki bounded. Then, for some $\varepsilon>0$, given any finite subset $F$ of $X$ and any $m \in \mathbb{N}$, there exits $y \in X$ such that $y \notin B_{\rho}^{m}(x, \varepsilon)$ for every $x \in F$. Let $F_{n}=\left\{x_{1}, \ldots, x_{n}\right\} \subset X(n \in \mathbb{N})$. By our assumption, we can choose $y_{n} \in X$ such that $y_{n} \notin B_{\rho}^{m}\left(x_{k}, \varepsilon\right)$ $(k=1, \ldots, n)$. Hence, the sequence $\left(y_{n}\right)$ formed in this way cannot have a left $\rho$-Bourbaki Cauchy subsequence which is a contradiction.

Although the sequence in Example 3.17 is not $\rho$-convergent, the subsequence $\left(\frac{1}{n}\right)$ of $\left(x_{n}\right)$ is $\rho$ convergent to zero. This means that if weakly left $K$-(cofinally) Bourbaki Cauchy or left $\rho$-(cofinally) Bourbaki Cauchy sequence has a $\rho$-convergent subsequence, then the sequence itself is not need to be $\rho$-convergent. By virtue of this fact, we define left $\rho$-(cofinally) Bourbaki completeness and weakly left $K$-(cofinally) Bourbaki completeness in the following way.

Definition 3.24. A quasi metric space $(X, \rho)$ is said to be left (right) $\rho$-(cofinally) Bourbaki complete if every left (right) $\rho$-(cofinally) Bourbaki Cauchy sequence has a $\rho$-convergent subsequence. It is said to be weakly left (right) $K$-(cofinally) Bourbaki complete if every weakly left (right) $K$-(cofinally) Bourbaki Cauchy sequence has a $\rho$-convergent subsequence. It is said to be $\rho^{s}$-(cofinally) Bourbaki complete if every $\rho^{s}$-(cofinally) Bourbaki Cauchy sequence has a $\rho$-convergent subsequence. 
We have the following diagram which is also true for right notions.

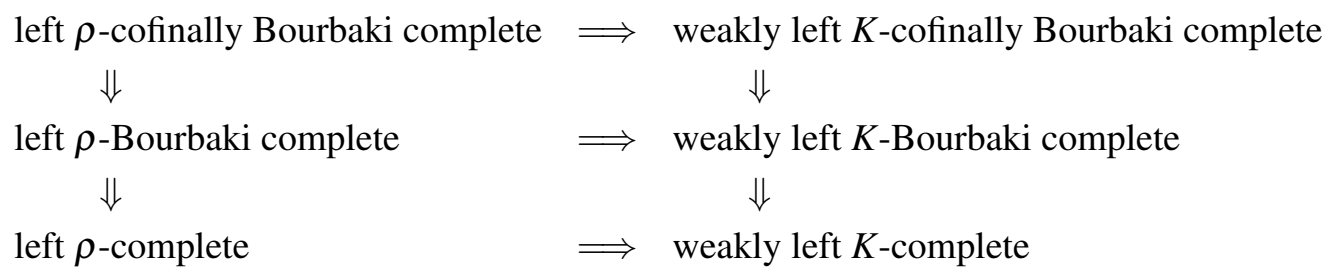

The following example shows that weakly left $K$-(cofinally) Bourbaki completeness of a quasi metric space does not imply that it is left $\rho$-(cofinally) Bourbaki complete.

Example 3.25. Let $X=\mathbb{N}$ and let $\rho$ be the quasi metric on $X$ defined as

$$
\rho(r, s)=\left\{\begin{array}{lc}
0, & \text { if } r=s, \\
\frac{1}{r}, & \text { if } s>r, s \text { even, } r \text { odd }, \\
1, & \text { otherwise. }
\end{array}\right.
$$

$(X, \rho)$ is weakly left $K$-(cofinally) Bourbaki complete since weakly left $K$-(cofinally) Bourbaki Cauchy sequences are only trivial one. Consider the sequence $\{2,4,6, \ldots\}$ which is left $\rho$-Bourbaki Cauchy but it has no $\rho$-convergent subsequence. Hence, $(X, \rho)$ is not left $\rho$-(cofinally) Bourbaki complete.

Note that left $\rho$-(cofinally) Bourbaki completeness or weakly left $K$-(cofinally) Bourbaki completeness of a quasi metric space does not imply that it is right $\rho$-(cofinally) Bourbaki complete or weakly right $K$-(cofinally) Bourbaki complete and vice versa. We observe that the subspace $Z$ in Example 3 of [10] is left $\rho$-(cofinally) Bourbaki complete and so it is weakly left $K$-(cofinally) Bourbaki complete since the terms of a left $\rho$-(cofinally) Bourbaki Cauchy sequence in this space are constant after a definite term. But, since $Z$ is not weakly right $K$-complete, it is neither weakly right $K$-(cofinally) Bourbaki complete nor right $\rho$-(cofinally) Bourbaki complete.

It is obvious that if a quasi metric space is weakly left (right) $K$-(cofinally) Bourbaki complete, then it is $\rho^{s}$-(cofinally) Bourbaki complete. But, $\rho^{s}$-(cofinally) Bourbaki completeness does not imply that the space is both weakly left $K$-(cofinally) Bourbaki complete and weakly right $K$-(cofinally) Bourbaki complete. According to the Example 4 in [10], $X$ is not weakly left $K$-(cofinally) Bourbaki complete and weakly right $K$-(cofinally) Bourbaki complete since $X$ is not weakly left $K$-complete and weakly right $K$-complete.

Theorem 3.26. Every weakly left $K$-Bourbaki complete quasi metric space is weakly left $K$-complete.

Proof. Let $(X, \rho)$ be a weakly left $K$-Bourbaki complete quasi metric space and $\left(x_{n}\right)$ be a left $K$-Cauchy sequence in $X$. Then, $\left(x_{n}\right)$ is also a weakly left $K$-Bourbaki Cauchy sequence and so it has a $\rho$-convergent subsequence. From Proposition 1.2.4 in [23], $\left(x_{n}\right)$ is $\rho$-convergent to the same point which implies that $X$ is left $K$-complete or equivalently it is weakly left $K$-complete.

Similarly, we have the following result for completeness.

Theorem 3.27. In a $T_{1}$ quasi metric space $(X, \rho)$, if $\lambda=\sup _{\substack{x, y \in X \\ x \neq y}} \frac{\rho(x, y)}{\rho(y, x)}$ exists, then the following statements are equivalent.

(1) $X$ is left $\rho$-(cofinally) Bourbaki complete (weakly left $K$-(cofinally) Bourbaki complete). 
(2) $X$ is right $\rho$-(cofinally) Bourbaki complete (weakly right $K$-(cofinally) Bourbaki complete).

(3) $X$ is $\rho^{s}$-(cofinally) Bourbaki complete.

On the contrary to the metric case, in a quasi metric space, Bourbaki boundedness cannot be characterized in terms of Bourbaki Cauchy sequences. In the quasi metric case, if $X$ is hereditarily $\rho$-Bourbaki bounded set, then we can characterize it with weakly left $K$-Bourbaki Cauchy sequences. We call a set as hereditarily $\rho$-Bourbaki bounded if every subset of $X$ is $\rho$-Bourbaki bounded.

Theorem 3.28. Let $(X, \rho)$ be a quasi metric space. Then the following statements are equivalent.

(1) $X$ is hereditarily $\rho$-Bourbaki bounded.

(2) Every countable subset of $X$ is $\rho$-Bourbaki bounded.

(3) Every sequence in $X$ has a weakly left K-Bourbaki Cauchy subsequence.

Proof. $1 \Rightarrow 2$ It is obvious.

$2 \Rightarrow 3$ Put $Z=\left\{x_{n}: n \in \mathbb{N}\right\}$ for an arbitrary sequence $\left(x_{n}\right)$ in $X$. By hypothesis, there exist a finite subset $F_{1} \subset Z$ and $m_{1} \in \mathbb{N}$ such that $Z \subset \cup\left\{B_{\rho}^{m_{1}}(x, 1): x \in F_{1}\right\}$. For a $n_{1} \in \mathbb{N}$, let $x_{n_{1}} \in F_{1}$ and $M_{1}=\{n \in$ $\left.\mathbb{N}: n \geq n_{1}, x_{n} \in B_{\rho}^{m_{1}}\left(x_{n_{1}}, 1\right)\right\} \subset \mathbb{N}$. The set $Z_{1}=\left\{x_{n}: n \in M_{1}, n>n_{1}\right\}$ is also $\rho$-Bourbaki bounded. Then there exist a finite subset $F_{2} \subset Z_{1}$ and $m_{2} \in \mathbb{N}$ such that $Z_{1} \subset \cup\left\{B_{\rho}^{m_{2}}(x, 1 / 2): x \in F_{2}\right\}$. For a $n_{2} \in M_{1}$, let $x_{n_{2}} \in F_{2}$ and $M_{2}=\left\{n \in M_{1}: n \geq n_{2}, x_{n} \in B_{\rho}^{m_{2}}\left(x_{n_{2}}, 1 / 2\right)\right\} \subset M_{1}$. Since the set $Z_{2}=\left\{x_{n}: n \in M_{2}, n>n_{2}\right\}$ is also $\rho$-Bourbaki bounded, we continue the process in the same manner. Hence, we obtain infinite nested sets $\ldots \subset M_{2} \subset M_{1}$ and an increasing sequence $n_{1}<n_{2}<\ldots$, where $n_{k} \in M_{k}$ for all $k \in \mathbb{N}$. Given any $\varepsilon>0$ choose $k_{\varepsilon} \in \mathbb{N}$ such that $\frac{1}{k_{\varepsilon}}<\varepsilon$. It follows that due to the fact that $M_{j} \subset M_{k_{\varepsilon}}$ for every $j>k_{\varepsilon}$, $x_{n_{j}} \in B_{\rho}^{m_{k_{\varepsilon}}}\left(x_{n_{k_{\varepsilon}}}, \varepsilon\right)$. This implies that $\left(x_{n_{j}}\right)$ is weakly left $K$-Bourbaki Cauchy subsequence of $\left(x_{n}\right)$ which completes the proof.

$3 \Rightarrow 1$ On the contrary, we suppose that $Y$ is not $\rho$-Bourbaki bounded subset of $X$. Then there exists an $\varepsilon>0$. For $F_{1}=\left\{x_{1}\right\} \subset Y$ and arbitrary $m \in \mathbb{N}$, there is $x_{2} \in Y$ such that $x_{2} \notin B_{\rho}^{m}\left(x_{1}, \varepsilon\right)$. Likewise, for $F_{2}=\left\{x_{1}, x_{2}\right\} \subset Y$, there is $x_{3} \in Y$ such that $x_{3} \notin B_{\rho}^{m}\left(x_{1}, \varepsilon\right) \cup B_{\rho}^{m}\left(x_{2}, \varepsilon\right)$. In this manner, we obtain a sequence $\left(x_{n}\right)$ in $X$ such that $x_{n} \notin B_{\rho}^{m}\left(x_{k}, \varepsilon\right)$ for $n>k$. This contradicts the fact that every sequence in $X$ has a weakly left $K$-Bourbaki Cauchy subsequence.

Theorem 3.29. Let $(X, \rho)$ be a quasi metric space. If $X$ is right $\bar{\rho}$-Bourbaki complete, then the $\bar{\rho}$-closure of all $\rho$-Bourbaki bounded subset in $X$ is $\bar{\rho}$-sequentially compact.

Proof. Suppose that every left $\rho$-Bourbaki Cauchy sequence in $X$ has a $\bar{\rho}$-convergent subsequence. Let $B \subset X$ be a $\rho$-Bourbaki bounded subset in $X$. Then, Lemma 3.12 implies that $\tau_{\bar{\rho}}-\mathrm{Cl}(B)$ is $\rho$-Bourbaki bounded. Hence, by Theorem 3.22, given any sequence $\left(x_{n}\right)$ in $\tau_{\bar{\rho}}-\mathrm{Cl}(B)$, there exists a left $\rho$-Bourbaki Cauchy subsequence. By our assumption, it has a subsequence which is $\bar{\rho}$-convergent to some $x \in X$. Since $\tau_{\bar{\rho}}-\mathrm{Cl}(B)$ is $\bar{\rho}$-closed, we have $x \in \tau_{\bar{\rho}}-\mathrm{Cl}(B)$ which proves that the $\bar{\rho}$-closure of any $\rho$-Bourbaki bounded set is $\bar{\rho}$-sequentially compact.

Theorem 3.30. A compact quasi metric space is left $\rho$-Bourbaki complete (weakly left $K$-Bourbaki complete) and $\rho$-Bourbaki bounded.

Proof. Let $(X, \rho)$ be a compact quasi metric space. Hence $X$ is sequentially compact, that is, every sequence in $X$ has a $\rho$-convergent subsequence. Then it follows that the space is left $\rho$-Bourbaki complete 
(weakly left $K$-Bourbaki complete). From the fact that a $\tau_{\rho}$-compact quasi metric space is precompact, we see that it is also $\rho$-Bourbaki bounded.

Theorem 3.31. A $\rho$-Bourbaki bounded and left $\rho$-Bourbaki complete quasi metric space is sequentially compact.

Proof. If $(X, \rho)$ is $\rho$-Bourbaki bounded quasi metric space, from Theorem 3.22, every sequence in $X$ has a left $\rho$-Bourbaki Cauchy subsequence. By hypothesis, this subsequence has a $\rho$-convergent subsequence. Hence $X$ is sequentially compact.

One can easily have the following result.

Theorem 3.32. In a $T_{1}$ quasi metric space $(X, \rho)$, if $\lambda=\sup _{\substack{x, y \in X \\ x \neq y}} \frac{\rho(x, y)}{\rho(y, x)}$ exists, then the following statements are equivalent.

(1) $X$ is compact.

(2) $X$ is sequentially compact.

(3) $X$ is left $\rho$-Bourbaki complete and $\rho$-Bourbaki bounded.

(4) $X$ is weakly left $K$-Bourbaki complete and $\rho$-Bourbaki bounded.

Theorem 3.33. A hereditarily $\rho$-Bourbaki bounded and weakly left $K$-Bourbaki complete quasi metric space is sequentially compact.

Proof. Let $\left(x_{n}\right)$ be a sequence in $X$. From Theorem 3.28, it has a weakly left $K$-Bourbaki Cauchy subsequence. This subsequence has a $\rho$-convergent subsequence. Hence $X$ is sequentially compact.

We call a quasi metric space $(X, \rho)$ as uniformly locally compact if there exists $\delta>0$ such that the closure of $B_{\rho}(x, \delta)$ with respect to the topology $\tau_{\rho}$ is compact for all $x \in X$. Also, we call a quasi metric space $(X, \rho)$ as $\rho$-locally Bourbaki bounded if every point $x$ in $X$ has a $\rho$-Bourbaki bounded neighbourhood. Recall that $V \subset X$ is a neighbourhood of $x \in X$ if there exists $U \in \tau_{\rho}$ such that $x \in U \subset V$. By using these notions, we have the following results.

Lemma 3.34. If $B$ is a $\rho$-compact set in a uniformly locally compact quasi metric space $(X, \rho)$, then the set $\tau_{\rho}-C l\left(B^{\delta / 2}\right)$ is $\rho$-compact.

Proof. Suppose that there exists $\delta>0$ such that for each $x \in X, \tau_{\rho}-\operatorname{Cl}\left(B_{\rho}(x, \delta)\right)$ is $\rho$-compact. Let $B$ be a $\rho$-compact set in $X$. Then, the $\rho$-open cover $\left\{B_{\rho}\left(y, \frac{\delta}{2}\right): y \in B\right\}$ of $B$ has a finite subcover, that is, $B \subseteq \bigcup_{y \in F} B_{\rho}\left(y, \frac{\delta}{2}\right)$, where $F \subset B$ and $F$ is finite. The inclusion

$$
\left(B_{\rho}\left(y, \frac{\delta}{2}\right)\right)^{\delta / 2}=B_{\rho}^{2}\left(y, \frac{\delta}{2}\right) \subseteq \tau_{\rho}-\mathrm{Cl}\left(B_{\rho}(y, \delta)\right)
$$

implies that $\tau_{\rho}-\mathrm{Cl}\left(B_{\rho}\left(y, \frac{\delta}{2}\right)\right)^{\delta / 2}$ is $\rho$-compact as it is a $\rho$-closed subset of a $\rho$-compact set. It follows that

$$
B^{\delta / 2} \subseteq \bigcup_{y \in F}\left(B_{\rho}\left(y, \frac{\delta}{2}\right)\right)^{\delta / 2} \subseteq \bigcup_{y \in F} \tau_{\rho}-\operatorname{Cl}\left(B_{\rho}(y, \delta)\right) .
$$

So $\tau_{\rho}-\mathrm{Cl}\left(B^{\delta / 2}\right)$ is $\rho$-compact.

Theorem 3.35. A uniformly locally compact quasi metric space $(X, \rho)$ is $\rho$-locally Bourbaki bounded and left $\rho$-cofinally Bourbaki complete. 
Proof. Let $X$ be a uniformly locally compact quasi metric space. Then, there exists a $\delta>0$ such that, for each $x \in X, \tau_{\rho}-\mathrm{Cl}\left(B_{\rho}(x, \delta)\right)$ is $\rho$-compact neighbourhood of $x$ and so $\rho$-Bourbaki bounded neighbourhood of $x$. Hence, $X$ is $\rho$-locally Bourbaki bounded.

Now, let $\left(x_{n}\right)$ be a left $\rho$-cofinally Bourbaki Cauchy sequence in $X$. Then, there exist $m \in \mathbb{N}, x \in X$ and an infinite subset $\left\{n_{1}<n_{2}<\ldots<n_{k}<\ldots\right\}$ of $\mathbb{N}$ such that $x_{n_{k}} \in \tau_{\rho}-\operatorname{Cl}\left(B_{\rho}^{m}\left(x, \frac{\delta}{2}\right)\right)$ for all $k \in \mathbb{N}$. It follows that $\left(x_{n_{k}}\right)$ has a $\rho$-convergent subsequence since $\tau_{\rho}-\operatorname{Cl}\left(B_{\rho}^{m}\left(x, \frac{\delta}{2}\right)\right)$ is $\rho$-compact from the last lemma. Hence, we conclude that $X$ is left $\rho$-cofinally Bourbaki complete.

\section{CONCLUSION}

In a quasi metric space $(X, \rho)$, we can also define a sequence named as left $K$-Bourbaki Cauchy. A sequence $\left(x_{n}\right)$ in $X$ is said to be left $K$-Bourbaki Cauchy if, for every $\varepsilon>0$, there exist $n_{0} \in \mathbb{N}$ and $m \in \mathbb{N}$ such that $x_{n} \in B_{\rho}^{m}\left(x_{r}, \varepsilon\right)\left(x_{n} \in B_{\bar{\rho}}^{m}\left(x_{r}, \varepsilon\right)\right)$ for all $n \geq r \geq n_{0}$. This new notion of Bourbaki Cauchyness is not equivalent to other notions (weakly left $K$-Bourbaki Cauchy and left $\rho$-Bourbaki Cauchy). In fact, consider the quasi metric space in Example 3.15 and the sequence $\left(w_{n}\right)=\left(0,1, \frac{1}{2}, \ldots, \frac{1}{n}, \ldots\right)$. For all $n \in \mathbb{N}$, we have $\rho\left(w_{1}, w_{n}\right)=0$, which means that $\left(w_{n}\right)$ is weakly left $K$-Cauchy and so weakly left $K$-Bourbaki Cauchy. But, this sequence is not left $K$-Bourbaki Cauchy since we have $w_{s} \notin B_{\rho}^{m}\left(w_{r}, \frac{1}{2}\right)=\left[w_{r}, 1\right]$ for $s>r>1$ and $m \in \mathbb{N}$. Observe that these three types of Bourbaki Cauchyness are equivalent in a metric space. Similarly, one can define left $K$-Bourbaki completeness as every left $K$-Bourbaki Cauchy sequence has a $\rho$-convergent subsequence. In a quasi metric space $(X, \rho)$, the following statements are equivalent (see [32]):

(1) $X$ is compact.

(2) $X$ is left $K$-complete and precompact.

In a metric space $(X, d)$, it was proved in [20] that the following statements are equivalent

(1) $X$ is compact.

(2) $X$ is complete and precompact.

(3) $X$ is Bourbaki complete and Bourbaki bounded.

By virtue of these facts, we have an open problem for quasi metric spaces. We do not know whether the following statements are equivalent or not

(1) $X$ is compact.

(2) $X$ is left $K$-Bourbaki complete and $\rho$-Bourbaki bounded. Our second problem is that weakly left $K$-Bourbaki completeness and left $K$-Bourbaki completeness are equivalent or not.

\section{Acknowledgments}

The authors would like to thank the editor and anonymous referees for their valuable comments and suggestions.

\section{REFERENCES}

[1] W.A. Wilson, On quasi metric spaces, Amer. J. Math. 53 (1931), 675-684.

[2] G.E. Albert, A note on quasi metric spaces, Bull. Amer. Math. Soc. 47 (1941), 479-482.

[3] S. Bodjanova, Some basic notions of mathematical analysis in oriented metric spaces, Math. Slovaca 31(3) (1981), 277289.

[4] D. Doitchinov, On completeness in quasi-metric spaces, Topology Appl. 30(2) (1988), 127-148. 
[5] J. C. Kelly, Bitopological spaces, J. Proc. London Math. Soc. 13 (1963), 71-89.

[6] H.-P.A. Künzi, A note on sequentially compact quasi-pseudometric spaces, Monatsh. Math. 95 (1983), 219-220.

[7] H.-P.A. Künzi, Complete quasi-pseudo-metric spaces, Acta Math. Hungar. 59 (1-2) (1992), 121-146.

[8] H.-P.A. Künzi, Nonsymmetric topology, Bolyai Soc. Math. Studies Vol. 4 Proc. Szekszard conference (1993), $303-338$.

[9] H.-P.A. Künzi, Nonsymmetric distances and their associated topologies: about the origin of basic ideas in the area of asymmetric topology, Handbook of the History of General Topology, Kluwer Acad. Publ. Dordrecht 2001, 853-968.

[10] I.L. Reilly, P.V. Subrahmanyam, M.K. Vamanamurthy, Cauchy sequences in quasi-pseudo-metric spaces, Monatsh. Math. 93(127) (1982), 127-140.

[11] I.L. Reilly, M.K. Vamanamurthy, On oriented metric spaces, Math. Slovaca 34(3) (1984), 299-305.

[12] S. Romaguera, Left $K$-completeness in quasi metric spaces, Math. Nachr. 157 (1992), 15-23.

[13] S. Romaguera, A. Gutierrez, A note on Cauchy sequences in quasi pseudo metric spaces, Glas. Mat. Ser. III 21(41):1 (1986), 191-200.

[14] R.A. Stoltenberg, On quasi metric spaces, Duke Math. J. 36 (1969), 65-71.

[15] S. Romaguera, M. Schellekens, Quasi-metric properties of complexity spaces, Topology Appl. 98(1-3) (1999), 311-322.

[16] M. Schellekens, The Smyth completion: a common foundation for denotational semantics and complexity analysis. In Mathematical foundations of programming semantics (New Orleans, LA, 1995), volume 1 of Electron. Notes Theor. Comput. Sci., page 22 pp. (electronic). Elsevier, Amsterdam, 1995.

[17] M.S. Waterman, T.F. Smith, W.A. Beyer, Some biological sequence metrics, Advances in Math. 20(3) (1976), $367-387$.

[18] M. Atsuji, Uniform continuity of continuous functions of metric spaces, Pacific J. Math. 8 (1958), 11-16.

[19] S. Willard, General Topology, Dover Publications Inc., Mineola, NY, 2004.

[20] I. Garrido, A.S. Merono, New types of completeness in metric spaces Ann. Acad. Sci. Fenn. Math. 39 (2014), $733-758$.

[21] I. Garrido, A.S. Merono, Uniformly metrizable bornologies, J. Convex Anal. 20 (1) (2013), 285-299.

[22] I. Garrido, A.S. Merono, Two classes of metric spaces Appl. Gen. Topol. 17 (2016), 57-70.

[23] S. Cobzas, Functional Analysis in Asymmetric Normed Spaces, Birkhauser, 2012.

[24] Y-B. Shen, W. Zhao, Gromov pre-compactness theorems for nonreversible Finsler manifolds, Differential Geom. Appl. 28 (2010), 565-581.

[25] C. Alegre, I. Ferrando, L.M. García-Raffi, E. A. Sánchez Pérez, Compactness in asymetric normed spaces, Topology Appl. 155 (2008), 527-539.

[26] J.J. Conradie, M.D. Mabula, Completeness, precompactness and compactness in finite-dimensional asymmetrically normed lattices, Topology Appl. 160 (2013), 2012-2024.

[27] J.J. Conradie, M.D. Mabula, Convergence and left-K-sequential completeness in asymmetrically normed lattices, Acta Math. Hung. 139 (2013) 147-159.

[28] L.M. Garcia-Raffi, Compactness and finite dimension in asymmetric normed linear spaces, Topology Appl. 153 (2005) 844-853.

[29] L.M. Garcia-Raffi, S. Romaguera, E. A. Sanchez-Perez, Sequence spaces and asymmetric norms in the theory of computational complexity, Math. Comput. Model. 36 (2002), 1-11.

[30] S. Cobzas, Separation of convex sets and the best approximation in spaces with asymmetric norm, Quaest. Math. 27 (3) (2014), 275-296.

[31] S. Romaguera, M. Sanchis, Semi-Lipschitz functions and best approximation in quasi-metric spaces, J. Approx. Theory, 103 (2000), 292-301.

[32] H.-P.A. Künzi, M. Mrsevic, I.L. Reilly, M. K. Vamanamurthy, Convergence, precompactness and symmetry in quasiuniform spaces, Math. Japon. 38 (1993), 239-253. 